\title{
Localization of the Invadopodia-Related Proteins Actinin-1 and Cortactin to Matrix-Contact-Side Cytoplasm of Cancer Cells in Surgically Resected Lung Adenocarcinomas
}

\author{
Shinichi Hirooka ${ }^{\text {a }}$ Takumi Akashi $^{\text {a }}$ Noboru Ando $^{\text {a }}$ Yoshimi Suzuki $^{a}$ \\ Noriko Ishida ${ }^{a}$ Morito Kurata $^{a}$ Touichirou Takizawa $^{a}$ Kou Kayamori $^{b}$ \\ Kei Sakamoto ${ }^{b}$ Naoyuki Fujiwara ${ }^{c}$ Masao Kojima ${ }^{c}$ Yoshinobu Eishi ${ }^{a}$ \\ Departments of ${ }^{\text {P Pathology, }}{ }^{\mathrm{b}}$ Oral Pathology and ${ }^{\mathrm{C}}$ Thoracic Surgery, Tokyo Medical and Dental University, \\ Tokyo, Japan
}

\section{Key Words}

Lung $\cdot$ Adenocarcinoma $\cdot$ Actinin-1 $\cdot$ Cortactin $\cdot$

Basement membrane $\cdot$ Laminin $\cdot$ Invadopodia $\cdot$ Ect2

\begin{abstract}
Objectives: Actin-associated proteins at cell-matrix-contact sites form invadopodia in cancer cells and participate in migration, matrix degradation and invasion. We investigated an alteration of subcellular localization of invadopodia-related actin-associated proteins, actinin-1 and cortactin, in lung adenocarcinomas, its clinical significance, and its possible regulatory factors. Methods: Invadopodia-related proteins, actinin-1 and cortactin, were immunohistochemically examined in 90 cases of lung adenocarcinomas. Expression of invadopodia-associated proteins and their possible regulators in lung adenocarcinomas were examined by real-time RTPCR, database search, and immunohistochemistry. Results: Actinin-1 and cortactin showed matrix-contact-side localization in adenocarcinoma cells, but rarely in normal bronchiolar epithelial cells, alveolar cells, or precursor lesion atypical adenomatous hyperplasia cells. Immunoelectron-micro-
\end{abstract}

scopic examination of adenocarcinoma cells revealed actinin-1 localization to matrix-contact-side cytoplasm with cytoplasmic protrusions. Matrix-contact-side localization of actinin-1 and cortactin was correlated with tumor stages, lymph node metastasis, vascular permeation, and loss of basement membrane. The tumor-specific survival rate was worse for the group in which matrix-contact-side localization of cortactin was high than for the low group. mRNA of the Rho guanine exchange factor epithelial cell transforming sequence-2 (Ect2) tended to be overexpressed in lung adenocarcinomas and cytoplasmic expression of Ect2 tended to be correlated with matrix-contact-side localization of actinin-1. Conclusion: Matrix-contact-side localization of invadopodia-related proteins in the lung adenocarcinoma cells were correlated with invasion, metastasis, and poor prognosis. Ect2 was a possible regulator of matrix-contact-side localization of invadopodia-related proteins.

Copyright $\odot 2011$ S. Karger AG, Basel

S.H. and T.A. contributed equally to this work.

\section{KARGER}

Fax +4161306 1234

E-Mail karger@karger.ch

www.karger.com
C 2011 S. Karger AG, Basel

1015-2008/11/0781-0010\$38.00/0

Accessible online at:

www.karger.com/pat
Takumi Akashi

Department of Pathology, Tokyo Medical and Dental University

1-5-45, Yushima, Bunkyo-ku

Tokyo 113-8519 (Japan)

Tel. +8135803 5661, Fax +8135803 0174, E-Mail akashi.path@tmd.ac.jp 
Actin-associated proteins form adhesive complexes with integrin receptors that connect the intracellular actin cytoskeleton with the extracellular matrix [1]. In the initial step of migration, moving cells form cytoplasmic protrusions at the front side's leading edge, where many matrix adhesive complexes are newly formed and actin filaments are elongated; actin-associated proteins at matrix-contact sites play a central role in the regulation of migration [2]. In cultured cancer cells, these actin-associated proteins, together with membrane-type matrix metalloproteinases (MT-MMPs), form invadopodia-specialized cytoplasmic protrusions from the ventral side to the underlying matrix [3]. A number of reports have shown degradation of the extracellular matrix that was localized to front-side cytoplasmic protrusions and ventral invadopodia [4-7]. Accordingly, it is possible that localization of actin-associated proteins at the matrix-contact-side cytoplasm of invasive cancer cells probably increases and participates in invasion through exaggerated migration and extracellular matrix degradation.

Previous studies have revealed abnormalities of invadopodia-related actin-associated proteins in human cancer tissues, including the overexpression of cortactin in breast, laryngeal and gastric cancers $[8,9]$, fascin in gastric and cholangiocarcinoma $[9,10]$, and vasodilatorstimulated phosphoprotein (VASP), actin-regulatory protein 2 (ARP2) and Wiscott-Aldrich syndrome protein family verprolin-homologous protein 2 (WAVE2) in lung adenocarcinomas [11, 12]. However, subcellular localization of these proteins to the matrix-contact-side cytoplasm, where they participate in adhesion to the extracellular matrix and degradation of it, has not been fully described in previous studies.

In this research, we studied the alteration of subcellular localization of invadopodia-related proteins, actinin-1, VASP, cortactin, and ARP2, in lung adenocarcinomas to elucidate a correlation with invasion and metastasis. We found that actinin-1, cortactin, and VASP showed localization to the matrix-contact-side cytoplasm facing surrounding mesenchymal tissues in lung adenocarcinoma cells. To confirm the subcellular localization of actinin-1 in the adenocarcinoma cells, we studied cancer cells by immunoelectron microscopy.

In lung adenocarcinomas, the normal structures of the alveolar and bronchiolar extracellular matrix, basement membrane, are often disrupted or absent and this significantly correlates with metastasis, prognosis, and tumor-associated stroma formation [13-16]. Accordingly, basement membrane disruption is considered as one of the important processes of lung cancer progression. In addition to overexpression of MMPs in cancer cells and/ or stromal cells [17-20], invadopodia-related proteins at matrix-contact side may also participate in the degradation of the basement membrane. Therefore, we examined the correlation between loss of basement membrane and matrix-contact-side localization of invadopodia-related proteins.

Assembly and turnover of invadopodia are affected by invadopodia-related actin-associated proteins and signal transduction regulators including kinases such as c-src, growth factors such as epidermal growth factor, and Rho family GTPases [21, 22]. Accordingly, it is possible to hypothesize that expression levels of the invadopodia-related proteins or the transduction regulators in the lung adenocarcinoma cells might regulate subcellular localization of invadopodia-related proteins to matrix-contact-side cytoplasm. Therefore, we examined mRNAs quantities of the invadopodia-related proteins, actinin-1, cortactin, VASP, fascin, ARP2, ARP3, and WAVE2; positive regulators of invadopodia assembly through c-src modulation, calpain 2 and protein tyrosine phosphatase 1B [23], and activators of Rho family GTPases, Rho guanine exchange factors (Rho GEF).

\section{Patients and Methods}

Patients (table 1)

Ninety cases of adenocarcinomas and 9 lesions of atypical adenomatous hyperplasia (AAH) were examined. Each lesion was surgically resected in the Tokyo Medical and Dental University Hospital between 1997 and 2007, fixed in formalin and embedded in paraffin. In addition, another 27 cases of fresh adenocarcinoma specimens were embedded in OCT compound (Tissue Tek, Torrance, Calif., USA) and frozen in liquid nitrogen. Each patient underwent lobectomy or segmentectomy with lymph node dissection. No patient underwent preoperative chemotherapy. Informed consent to the use of surgical materials for research was obtained from each patient.

\section{Histological Evaluation}

Adenocarcinomas were classified into papillary, acinar, solid, and bronchioloalveolar subtypes according to WHO classification criteria [24]. Mixed subtypes were represented by the most dominant subtype among the papillary, acinar, or solid ones [25]. One representative block of each case was used for the immunohistochemical studies. Tumor stages were determined according to the staging manual of International Association of the Study of Lung Cancer [26].

\section{Immunohistochemical Examination}

Antibodies used were as follows: actinin-1 (Sigma, St. Louis, Mo., USA; BM75.2, monoclonal), cortactin (Epitomics, Burlingame, Calif., USA; EP1922Y, monoclonal), VASP (Alexis Bio- 
chemicals, San Diego, Calif., USA; IE273, monoclonal), laminin-511/C1 (Santa Cruz Biotechnology, Santa Cruz, Calif., USA; D-18, monoclonal), ARP2 (Santa Cruz Biotechnology; goat polyclonal, K-15) [12], MT1-MMP (Daiichi Fine Chemicals, Takaoka, Japan; 114-6G6, monoclonal), $\beta$-actin (Epitomics; EP1123Y, monoclonal), Ect2 (Santa Cruz Biotechnology; H-300, rabbit polyclonal) [27], biotin-labeled anti-mouse IgG and IgM, rhodamine-labeled anti-mouse IgM and anti-rabbit IgG (Santa Cruz Biotechnology).

Deparaffinized sections were heated in an antigen retrieval buffer (Nichirei Bioscience, Tokyo, Japan) at $98^{\circ} \mathrm{C}$ for $40 \mathrm{~min}$ in a microwave (actinin-1, cortactin and VASP), heated in $1 \mathrm{mmol} / \mathrm{l}$ EDTA (pH 8.0) at $110^{\circ} \mathrm{C}$ for $10 \mathrm{~min}$ in an autoclave (ARP2) or reacted with protease for $30 \mathrm{~min}$ (laminin-511). The slides were then treated with methanol containing $0.3 \%(\mathrm{v} / \mathrm{v})$ hydrogen peroxide for $10 \mathrm{~min}$ and reacted with primary antibodies, $\mathrm{ABC}$ detection kit (Vector, Burlingame, Calif., USA), and diaminobenzidine in succession. Frozen sections were fixed with acetone and were used for immunostaining of Ect 2 and actinin-1 without antigen retrieval.

Because a proportion of positive cells showed the more prominent difference among the examined cases and was easier to be quantified than the strength of staining, the degree of immunohistochemical findings was graded according to the proportion of positive cells as follows: none as ' 0 ', less than $5 \%$ as ' 1 ', $5 \%$ to one third as ' 2 ', one third to two thirds as ' 3 '; two thirds or more as ' 4 '. Grades 0 and 1 were classified as low and $2-4$ as high.

In the fluorescence double staining for MT1-MMP and actinin-1, tissues were fixed with $4 \%$ paraformaldehyde overnight, immersed successively with PBS containing 10, 15, and $20 \%$ sucrose, and then frozen in liquid nitrogen. Sections of $5-\mu \mathrm{m}$ thickness were heated in an antigen retrieval buffer at $98^{\circ} \mathrm{C}$ for $40 \mathrm{~min}$, reacted with anti-MT1-MMP, biotin-labeled anti-mouse IgG, streptavidin-FITC (DAKO, Carpinteria, Calif., USA), anti-actinin-1, and rhodamine-labeled anti-mouse IgM in this order, and observed by BZ-8100 (Keyence, Osaka, Japan). In the fluorescence double staining for $\beta$-actin and actinin-1, deparaffinized sections were heated in a citrate buffer $(\mathrm{pH} 6.0)$ at $120^{\circ} \mathrm{C}$ for $20 \mathrm{~min}$, reacted with mixed anti-actin and anti-actinin- 1 antibodies, rhodamine-labeled anti-rabbit IgG, biotin-labeled anti-mouse IgM, and streptavidin-FITC in this order.

\section{Immunoelectron Microscopy}

Paraformaldehyde-fixed frozen sections were heated in an antigen retrieval buffer at $70^{\circ} \mathrm{C}$ for $2 \mathrm{~h}$, incubated with anti-actinin-1 antibody followed by ABC detection kit, postfixed with $2 \%$ glutaraldehyde for $5 \mathrm{~min}$, and visualized with diaminobenzidine The sections were fixed again with $4 \% \mathrm{OsO}_{4}$, dehydrated with ethanol, and embedded in Epon. Ultrathin sections were observed by a transmission electron microscope (H-7100; Hitachi, Tokyo, Japan).

\section{Preparation of RNA and Quantitative Assay by Real-Time RT-PCR}

Total RNA was extracted from frozen samples of normal tissues, cancer tissues, and cultured cells using RNeasy Mini Kit (Qiagen, Valencia, Calif., USA) and reverse transcribed by PrimeScript ${ }^{\circledR}$ RT-PCR Kit (Takara Bio, Otsu, Japan) according to the manufacturer's instructions. For quantitative PCR, TaqMan ${ }^{\circledR}$ Gene Expression primer/probes and PCR Master Mix Kit (Ap-
Table 1. Characteristics of examined patients

\begin{tabular}{lll}
\hline & & Number of patients \\
\hline Sex & Male & 48 \\
& Female & 42 \\
\hline pT factor & T1 & 40 \\
& T2 & 40 \\
& T3 & 10 \\
\hline pN factor & N0 & 57 \\
& N1 & 17 \\
& N2, N3 & 16 \\
\hline p stage & I & 54 \\
& II & 16 \\
& III & 20 \\
\hline Histological & BAC & 21 \\
subtype & Acinar adenocarcinoma & 12 \\
& Papillary adenocarcinoma & 36 \\
& Solid adenocarcinoma & 21 \\
\hline Prognosis & Dead of lung cancer & 26 \\
& Alive & 60
\end{tabular}

plied Biosystems, Foster City, Calif., USA) were used, and gene expression was analyzed by ABI Prism 7900HT (Applied Biosystems Japan Ltd., Tokyo, Japan). The intensity of the reaction was evaluated from the quantity of total RNA in A549 lung adenocarcinoma cells (ng) corresponding to the initial number of PCR cycles to reveal the linear increase in reaction intensity (threshold cycle) for each sample on a logarithmic standard curve. Data on the quantity of RNA (ng) for objective genes were normalized using the data for GAPDH in each sample. To identify the candidate Rho GEFs which correlate to actinin-1 subcellular localization, an open database resource of cDNA microarray analysis of lung adenocarcinoma patients was examined (https://caarraydb. nci.nih.gov/caarray/publicExperimentDetailAction.do?expId= 1015945236141280) [28]. Of the 442 patients, 31 cases with adenocarcinoma with metastasis who died of tumors within 36 months after operation and 44 cases without metastasis who survived more than 60 months after operation were selected. Expression of 48 members of Rho GEF genes were compared between the two groups.

Mutation Analysis of Epidermal Growth Factor Receptor Gene DNA was extracted from deparaffinized sections with lysis buffer (20 mM Tris, $1 \mathrm{~mm}$ EDTA, 0.5\% Tween 20, and $2 \mathrm{mg} / \mathrm{ml}$ proteinase $\mathrm{K}$ ) at $55^{\circ} \mathrm{C}$ overnight, and proteinase activity was inactivated by boiling for $10 \mathrm{~min}$. Exons 19 and 21 were amplified by PCR, and PCR fragments were directly sequenced by ABI3100 analyzer (Applied Biosystems Japan Ltd.) with BigDye Terminator Cycle Sequencing Kit (Applied Biosystems). Primers for both PCR and sequencing reactions were as follows: exon 19: forward primer: 5'-TGTCATAGGGACTCTGGATCC-3', reverse primer: 5'-AAAGCAGAAACTCACATCGAG-3'. Exon 21: forward primer: 5'-GACCGTCGCTTGGTGCAC-3', reverse primer: 5'-TGCATGGTATTCTTTCTCTTCCG-3'. 

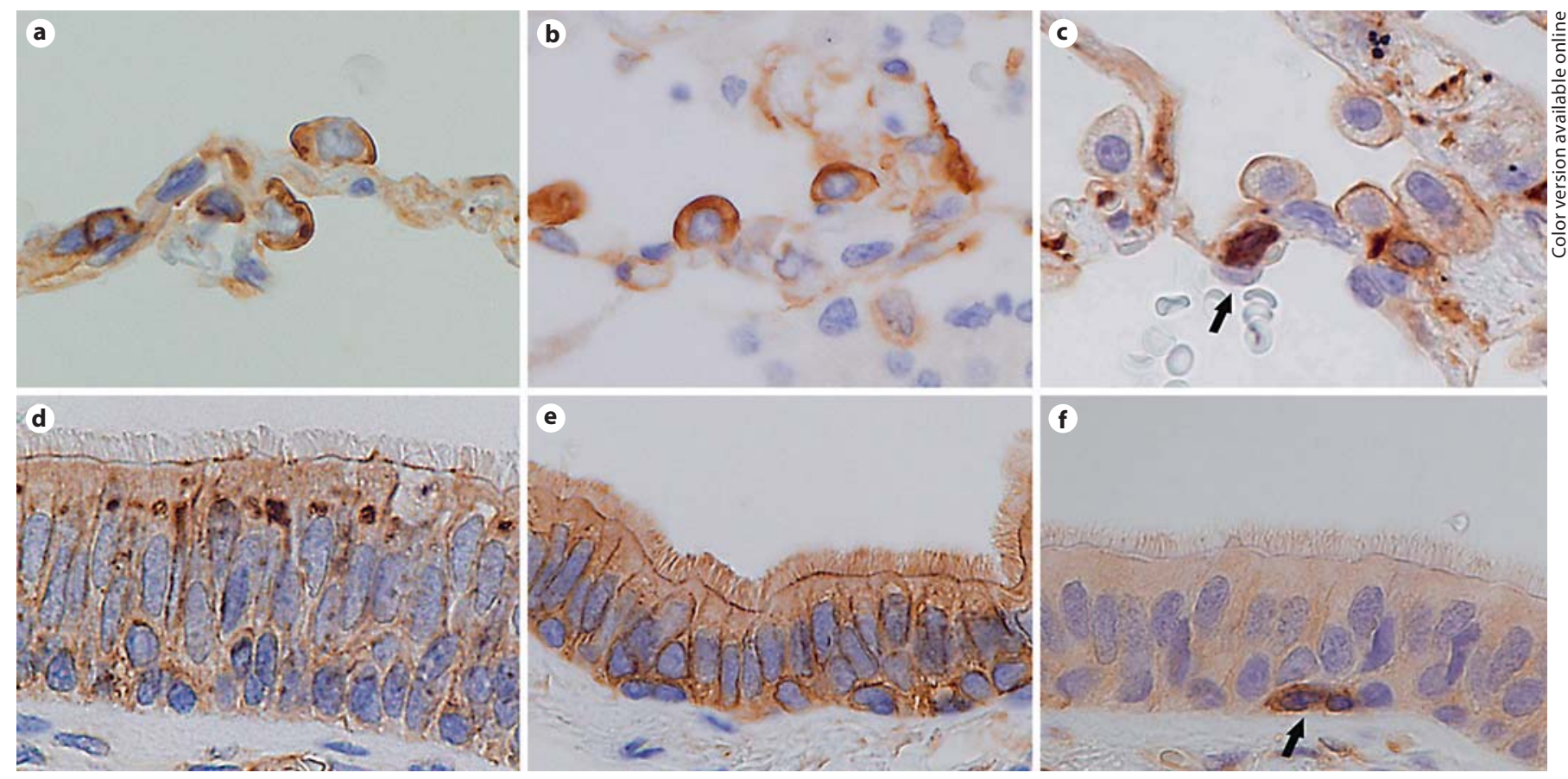

Fig. 1. Subcellular localization of actinin-1 (a, d), cortactin (b, e), and VASP (c, f) in normal alveolar $(\mathbf{a}-\mathbf{c})$ and bronchiolar $(\mathbf{d}-\mathbf{f})$ cells. Actinin-1 and cortactin are mainly localized in the apicaland intercellular-side cytoplasm of type II alveolar cells and bronchiolar cells and in the terminal bars of bronchiolar epithelial cells, where cilia adhere. Diffuse (actinin-1 and cortactin) and granular (actinin-1) cytoplasmic localization is also found. VASP is found in type II alveolar cells and inflammatory cells (arrow), but not in bronchiolar epithelial cells. Magnification: $\times 600$.

\section{Statistical Analysis}

Correlations between the patients' clinicopathological factors and immunohistochemical results were assessed by Mann-Whitney $U$ test. Tumor-specific survival curves were analyzed by the Kaplan-Meier method, and the statistical significance of differences was determined by log-rank test using StatFlex Ver. 6.0 statistical program (Artech, Osaka, Japan). Four patients who died of other diseases were excluded from the analysis of survival.

This study was approved by Internal Review Board of Bioethics of Tokyo Medical and Dental University.

\section{Results}

\section{Expression of Invadopodia-Related Proteins in}

Normal Lung Epithelial Cells and Adenocarcinoma

Cells

In normal type II alveolar cells and bronchiolar epithelium, actinin-1 showed localization to the apical- and intercellular-side cytoplasm in addition to diffuse and faint cytoplasmic distribution; however, matrix-contactside localization was rarely found. Actinin-1 was not detected in normal type I alveolar cells. Cortactin, another invadopodia-related protein, showed localization similar to actinin-1. VASP was also localized to the apical-side cytoplasm of type II alveolar cells but could not be detected in bronchiolar epithelium (fig. 1).

In adenocarcinoma cells, actinin-1 was frequently localized to the basal-side cytoplasm facing toward the surrounding interstitium as well as to the apical- and intercellular-side cytoplasm. Both cortactin and VASP showed matrix-contact-side localization similar to actinin-1 in adenocarcinoma cells (fig. 2a-e). Matrix-contact-side localization of VASP and cortactin were correlated with that of actinin-1; however, matrix-contact-side localization of VASP was less frequent than that of actinin-1 and cortactin (table 2). Basement membrane represented by laminin-511 was frequently disrupted or absent in adenocarcinomas (fig. 2f). Colocalization of MT1-MMP and actinin-1 at matrix-contact-side cytoplasm of adenocarcinoma cells was found focally in 2 of 5 adenocarcinoma cases by the fluorescence double stainings. Colocalization of actin and actinin- 1 at matrix-contact-side cytoplasm was also found (fig. 3). Cytoplasmic expression of ARP2 was subtle in most of the lung adenocarcinomas 

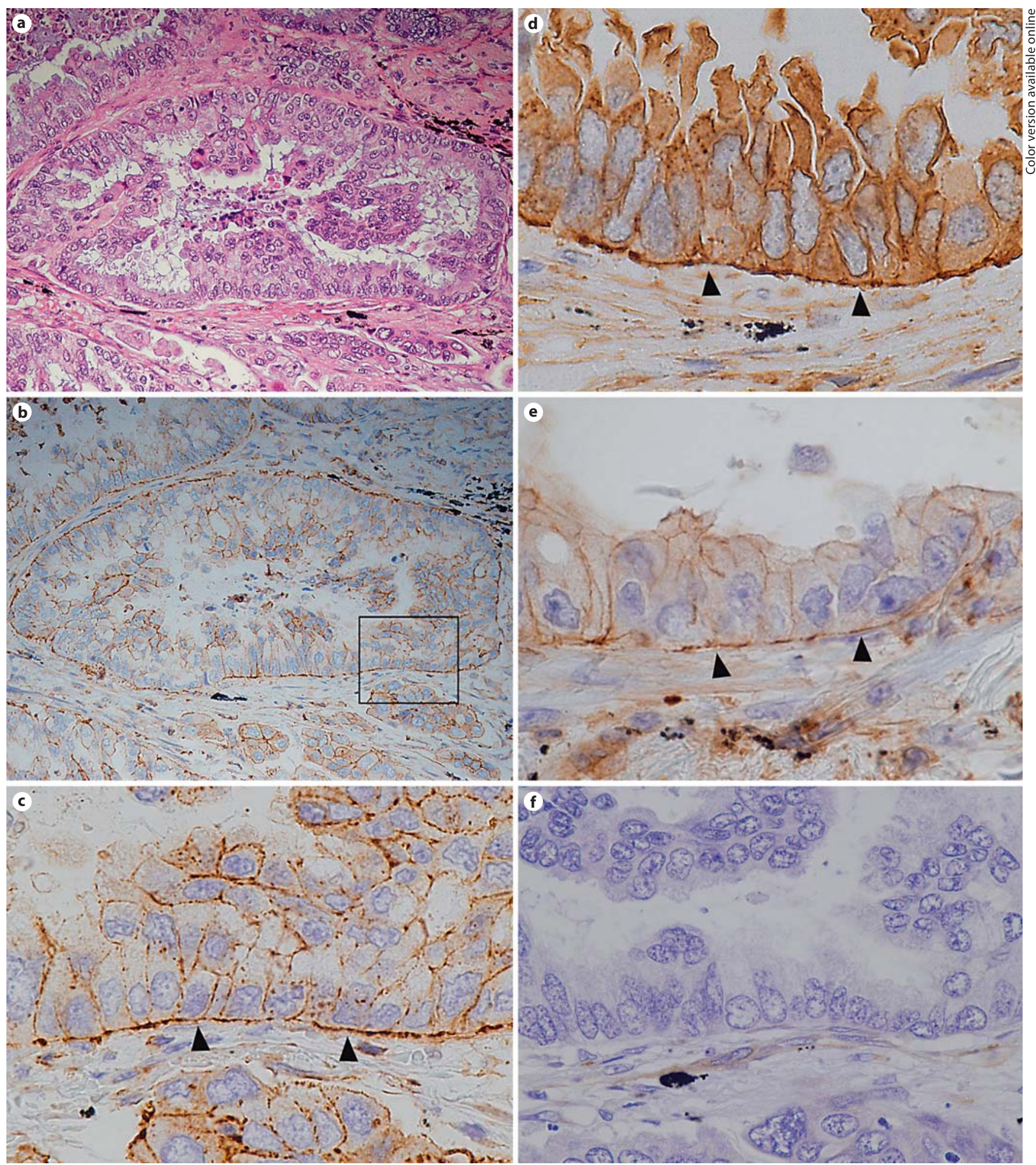

Fig. 2. Subcellular localization of actinin-1 (b, c), cortactin (d), $\operatorname{VASP}(\mathbf{e})$, and laminin-511 (f) in papillary adenocarcinoma. a Hematoxylin-eosin staining. Actinin-1 is localized to the matrixcontact-side cytoplasm of cancer cells in linear patterns (arrowheads) in addition to the apical- and intercellular-side cytoplasm.

Both cortactin and VASP are also found in the matrix-contactside (arrowheads), intercellular, and apical-side cytoplasm of cancer cells. Laminin-511 is absent around the cancer cells. Magnification: $\times 100(\mathbf{a}, \mathbf{b}) ; \times 400(\mathbf{c}-\mathbf{f})$. 
Table 2. Correlation between degree of matrix-contact-side localization of actinin-1 and clinicopathological parameters

\begin{tabular}{|c|c|c|c|}
\hline & \multicolumn{3}{|l|}{ Actinin $-1^{\mathrm{a}}$} \\
\hline & low & high & $\mathrm{p}$ \\
\hline $\begin{array}{l}\text { Tumor size, } \mathrm{cm} \\
\text { p stage }\end{array}$ & $2.7 \pm 1.7$ & $3.1 \pm 1.3$ & $0.09^{\mathrm{b}}$ \\
\hline I & 34 & 20 & $<0.001^{\mathrm{c}}$ \\
\hline II & 2 & 14 & \\
\hline III & 6 & 14 & \\
\hline \multicolumn{4}{|l|}{$\mathrm{pN}$ status } \\
\hline N0 & 36 & 21 & $<0.001^{\mathrm{c}}$ \\
\hline N1 & 3 & 14 & \\
\hline $\mathrm{N} 2, \mathrm{~N} 3$ & 3 & 13 & \\
\hline \multicolumn{4}{|c|}{ Lymphatic permeation } \\
\hline- & 28 & 15 & $<0.001^{\mathrm{c}}$ \\
\hline+ & 14 & 33 & \\
\hline \multicolumn{4}{|c|}{ Vascular permeation } \\
\hline- & 30 & 17 & $<0.001^{\mathrm{c}}$ \\
\hline+ & 12 & 31 & \\
\hline \multicolumn{4}{|l|}{ Cortactin $^{\mathrm{a}}$} \\
\hline Low & 32 & 13 & $<0.001^{\mathrm{c}}$ \\
\hline High & 10 & 35 & \\
\hline \multicolumn{4}{|l|}{ VASPa } \\
\hline Low & 22 & 19 & $<0.001^{\mathrm{c}}$ \\
\hline High & 3 & 17 & \\
\hline \multicolumn{4}{|l|}{ Ect $2^{d}$} \\
\hline Low & 13 & 3 & $0.052^{\mathrm{c}}$ \\
\hline High & 5 & 6 & \\
\hline \multicolumn{4}{|l|}{ EGFR mutation } \\
\hline- & 16 & 24 & $0.45^{\mathrm{c}}$ \\
\hline+ & 7 & 16 & \\
\hline
\end{tabular}

${ }^{a}$ Matrix-contact-side localization. ${ }^{b}$ Welch's t test. ${ }^{c}$ MannWhitney U test. ${ }^{\mathrm{d}}$ Examined with frozen sections.

and matrix-contact-side localization of ARP2 was found in only 3 of 77 adenocarcinoma cases (online suppl. fig. 1, www.karger.com/doi/10.1159/000322734).

\section{Immunoelectron-Microscopic Findings (fig. 4)}

Immunoelectron microscopy showed that actinin-1 was localized to the intercellular-side cytoplasm of bronchiolar cells, but matrix-contact-side localization was rarely found. The matrix-contact-side surface of normal bronchiolar cells was smooth and covered with linear basal lamina, with a thickness of $70 \mathrm{~nm}$. In the peripheral area of the papillary adenocarcinoma, basal lamina still covered the matrix-contact-side surface of cancer cells; however, the surface was irregular and showed cytoplasmic protrusions approximately $0.3 \mu \mathrm{m}$ in diameter and $1.5 \mu \mathrm{m}$ in length. Actinin-1 was found in the matrix-
Table 3. Correlation between degree of matrix-contact-side localization of cortactin and clinicopathological parameters

\begin{tabular}{llll}
\hline & \multicolumn{2}{l}{ Cortactin $^{\mathrm{a}}$} & \\
\cline { 2 - 4 } & low & high & $\mathrm{p}$ \\
\hline $\begin{array}{l}\text { Tumor size, cm } \\
\text { p stage }\end{array}$ & $2.7 \pm 1.7$ & $3.1 \pm 1.3$ & $0.022^{\mathrm{b}}$ \\
$\quad$ I & 33 & 21 & $0.024^{\mathrm{c}}$ \\
II & 4 & 12 & \\
$\quad$ III & 8 & 12 & \\
pN status & & & \\
$\quad$ N0 & 35 & 22 & $0.017^{\mathrm{c}}$ \\
$\quad$ N1 & 5 & 12 & \\
$\quad$ N2, N3 & 5 & 11 & \\
Lymphatic permeation & & & \\
$\quad-$ & 32 & 11 & $<0.001^{\mathrm{c}}$ \\
$\quad+$ & 13 & 34 & \\
Vascular permeation & & & \\
$\quad-$ & 35 & 12 & $<001^{\mathrm{c}}$ \\
$\quad+$ & 10 & 33 & \\
\hline
\end{tabular}

${ }^{a}$ Matrix-contact-side localization. ${ }^{b}$ Welch's t test. ${ }^{c}$ MannWhitney U test.

contact-side cytoplasm with cytoplasmic protrusions as well as in the intercellular-side cytoplasm.

\section{Relationship between Degree of Matrix-Contact-Side}

Localization of Actinin-1 and Histological Subtypes,

Basement Membrane Preservation, and

\section{Clinicopathological Findings}

Matrix-contact-side localization of actinin-1, cortactin and VASP was rarely found in AAH, a precursor lesion of adenocarcinomas, and was occasionally found in noninvasive bronchioloalveolar adenocarcinomas (BAC). The basement membrane component laminin-511 was well preserved in both AAH and BAC (fig. 5). The degree of actinin-1 matrix-contact-side localization was significantly higher in invasive papillary and solid adenocarcinomas than in noninvasive BAC. In contrast, loss of basement membrane was more prominent in papillary and solid adenocarcinomas. The degree of matrix-contactside localization of actinin-1 was reversely correlated with the degree of laminin-511 preservation. The degree of matrix-contact-side localization of cortactin showed similar results (fig. 6).

Forty-two of 69 invasive papillary, acinar or solid adenocarcinomas were accompanied with the component of noninvasive bronchioloalveolar subtype showing le- 
Fig. 3. Fluorescence double staining of actinin-1 and MT1-MMP (a) and actinin-1 and actin (b) in papillary adenocarcinoma. They are partly colocalized (arrowheads) at matrix-contact-side cytoplasm of cancer cells. Magnification: $\times 600$.
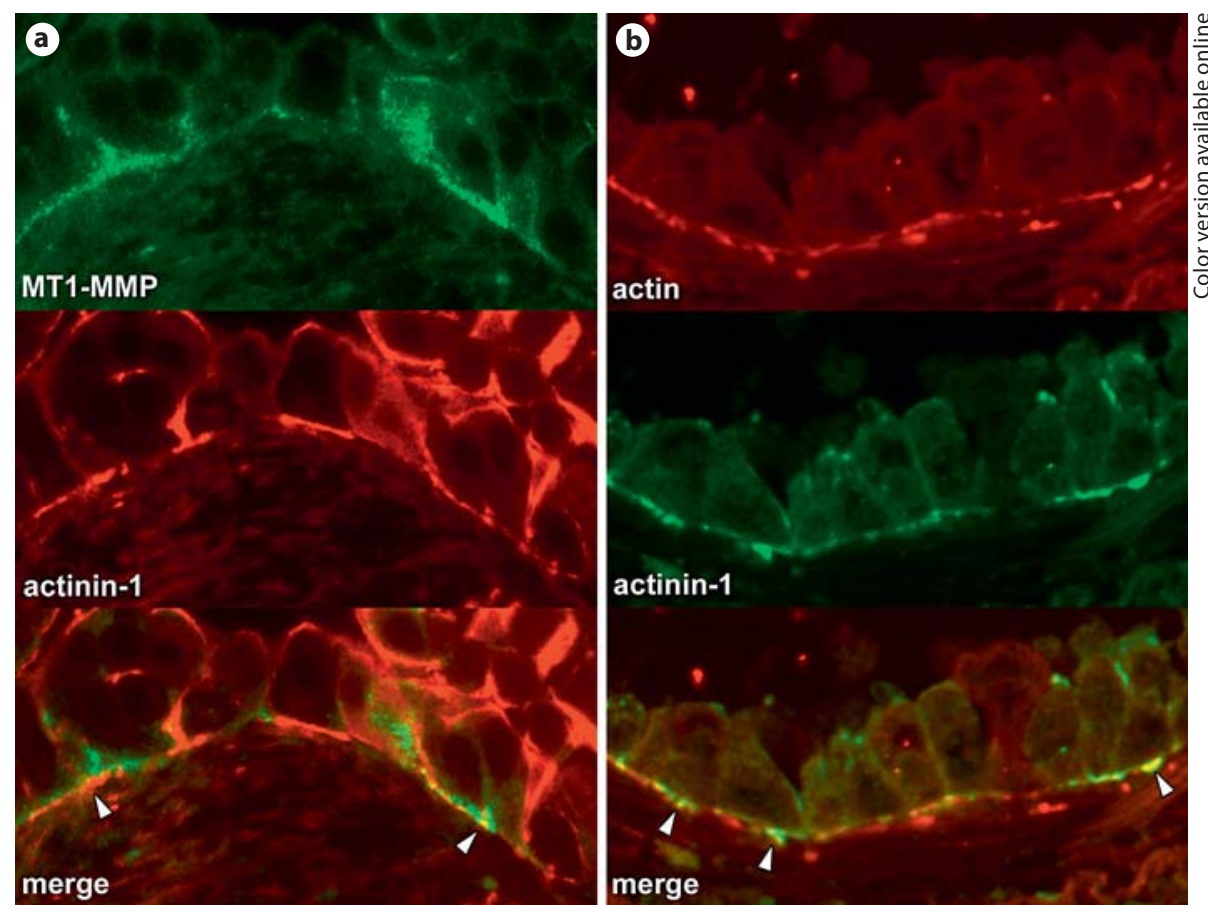

Table 4. mRNA expression of actin-associated and invadopodia regulatory molecules in normal lung and adenocarcinoma tissues by real-time PCR

pidic growth and well-preserved basement membrane. The degree of matrix-contact-side localization of both actinin-1 and cortactin was significantly lower in the noninvasive bronchioloalveolar component than in the invasive component (online suppl. fig. 2).

Forty-eight and 45 of 90 adenocarcinoma cases were estimated to have a high degree of matrix-contact-side localization of actinin-1 and cortactin, respectively. The degree of matrix-contact-side localization of both actinin-1 (table 2) and cortactin (table 3) was correlated with tumor stages, lymph node metastasis, and vascular permeation. The tumor-specific survival rate was worse for the group in which matrix-contact-side localization of cortactin was high than for the low group. Matrix-contact-side localization of actinin-1 was not significantly correlated with prognosis (fig. 7). 


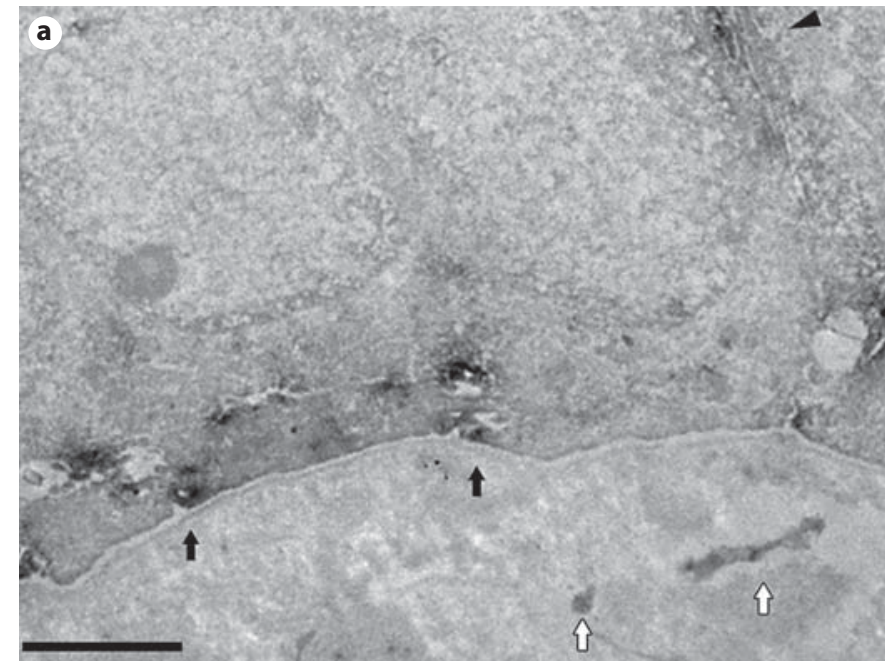

Fig. 4. Immunoelectron-microscopic findings of actinin-1 in normal bronchiolar cells and adenocarcinoma cells. a Normal bronchiolar cells. Actinin-1 mainly localizes to the intercellular-side cytoplasm (arrowhead) of bronchiolar cells, and matrix-contactside localization is scarcely found (closed arrows). Linea basal lamina, $70 \mathrm{~nm}$ thick, is found beneath the matrix-contact-side surface. $\mathbf{b}$ Cancer cells in the peripheral area of the papillary ad-

Expression of Ect2 and Its Relationship to Degree of

Matrix-Contact-Side Localization of Actinin-1

By the real-time PCR assay, the mRNAs of invadopodia-related proteins, actinin-1, cortactin, VASP, vinculin, fascin1, ARP2, ARP3, WAVE2, and positive regulators of invadopodia, calpain 2 and protein tyrosine phosphatase $1 \mathrm{~B}$ [23], were reduced in adenocarcinoma tissues compared to normal lung tissues (table 4). Because Rho GEFs are considered to be positive regulators of leading edge elongation and invadopodia assembly through Racl activation and subsequent actin remodeling [29], we screened the expression of Rho GEFs in lung adenocarcinomas using an open database resource of cDNA microarray analysis [28]. Among 48 members of both Dbl and DOCK families of Rho GEFs, Ect2 was the most possible Rho GEF overexpressed in adenocarcinomas with metastasis (online suppl. table 1). Although it was not statistically significant, Ect2 tended to be overexpressed in lung adenocarcinoma tissues rather than normal lung tissues by real-time RT-PCR (table 4). By the immunohistochemical study using frozen sections, cytoplasmic expression of Ect 2 was found in 11 of 27 adenocarcinomas and it tended to be correlated with the degree of matrix-contact-side localization of actinin-1 (fig. 8; table 2).

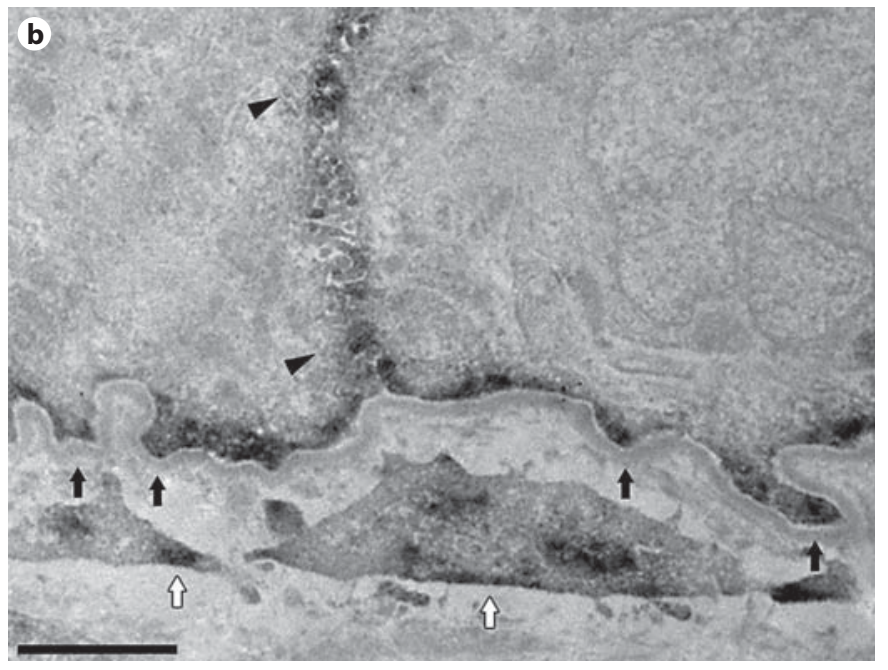

enocarcinoma. Actinin-1 localizes to the matrix-contact-side cytoplasm with cytoplasmic protrusions that are $0.3 \mu \mathrm{m}$ in diameter and $1.5 \mu \mathrm{m}$ in length (closed arrows). Actinin-1 also localizes to the intercellular-side cytoplasm (arrowheads). Basal lamina still remains beneath the matrix-contact-side surface of the cancer cells. Actinin-1 is also expressed in fibroblasts (open arrows). Bars: $2 \mu \mathrm{m}$.

Although EGF is a stimulator of both leading-edge elongation and invadopodia formation in cultured cells $[21,30], E G F R$-activating mutation did not correlate with matrix-contact-side localization of actinin-1 (table 2).

\section{Discussion}

We demonstrated that matrix-contact-side localization of actinin-1 and cortactin was preferentially observed in the lung adenocarcinoma cells. This intracytoplasmic localization was not apparent in precursor lesion adenomatous hyperplasia and less prominent in bronchioloalveolar-type adenocarcinomas which are considered to be a noninvasive subtype [25]. This localization was positively correlated with tumor stages, lymph node metastasis, and vascular permeation. These correlations suggest that matrix-contact-side localization of actinin-1 and cortactin participates in invasion and metastasis during the progression of lung adenocarcinomas.

Actinin-1 is expressed ubiquitously [31] and present in several types of adhesion sites, such as focal adhesions and hemidesmosomes at cell-matrix-contact sites and adherens junctions at cell-cell contact sites. Actinin-1 provides structural stability at adhesion sites by cross-link- 

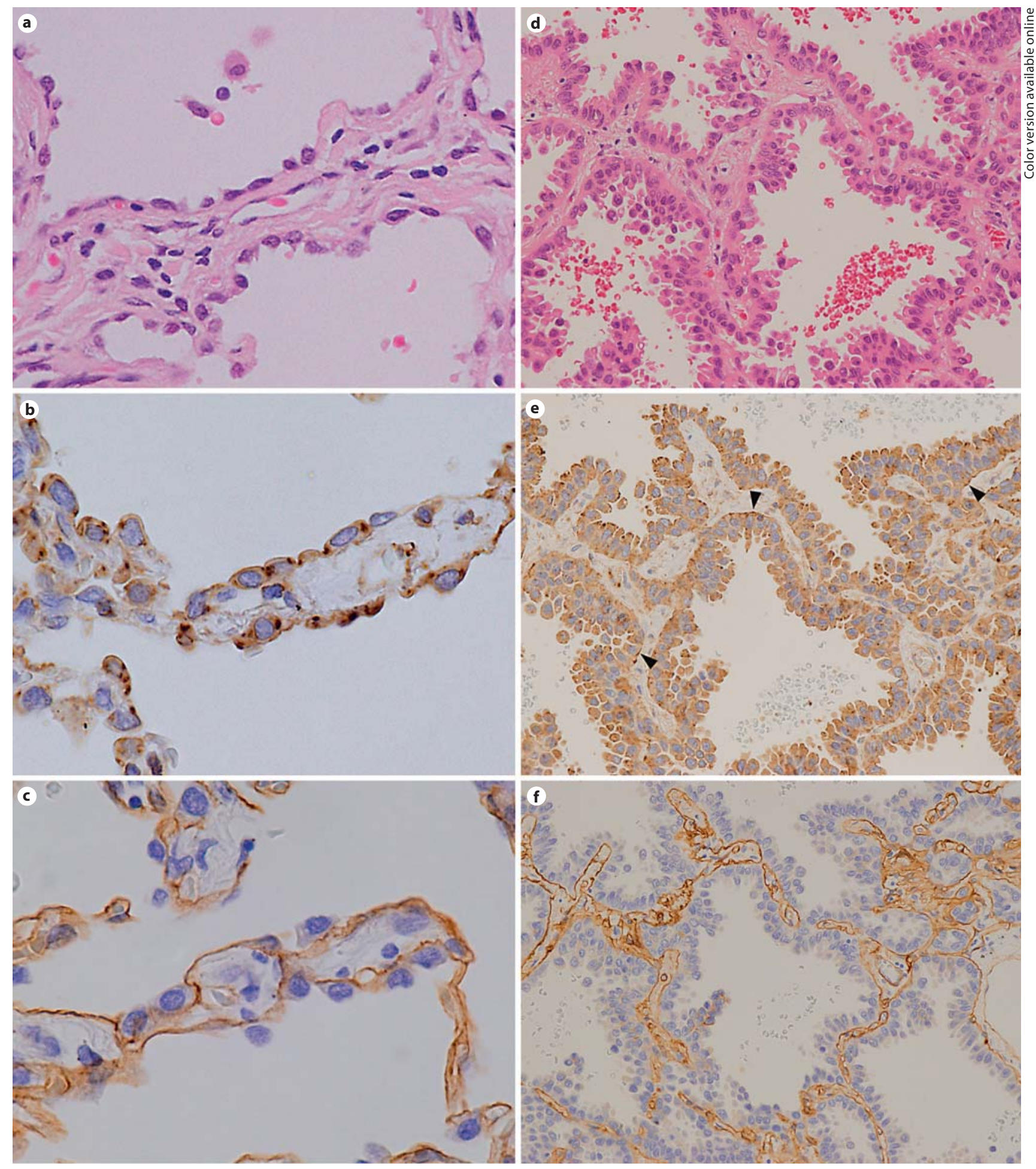

Fig. 5. Subcellular localization of actinin-1 (b, e) and laminin-511 $(\mathbf{c}, \mathbf{f})$ in AAH (a-c) and BAC (d-f). a, d Hematoxylin-eosin staining. Actinin-1 is found in the apical-side cytoplasm of both $\mathrm{AAH}$ and BAC cells. Granules of actinin-1 are also found in the cytoplasm of AAH cells. Matrix-contact-side localization of actinin-1 is not found in AAH but is occasionally found in BAC (arrowheads). Linear staining of laminin-511 is found in both AAH and $B A C$, which suggests a well-preserved basement membrane. Magnification: $\times 400(\mathbf{a}-\mathbf{c}) ; \times 100(\mathbf{d}-\mathbf{f})$. 

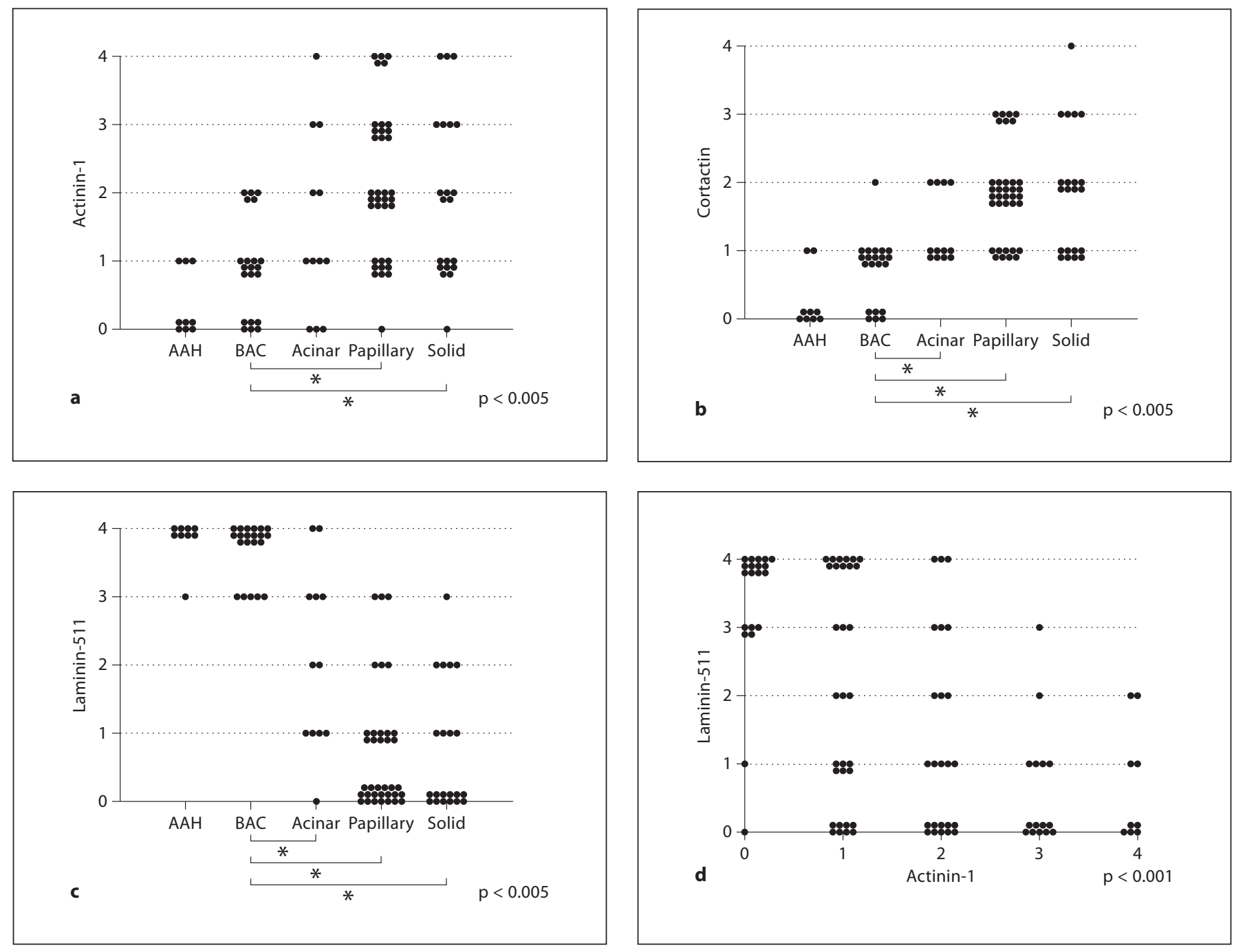

Fig. 6. Relationship between degree of matrix-contact-side actinin- 1 and cortactin localization, laminin-511 preservation, and histological subtypes. Relationship between histological subtypes of adenocarcinoma and degree of matrix-contact-side localization of actinin-1 (a) or cortactin (b). c Relationship between degree of laminin-511 and histological subtypes. Relationship between laminin-511 preservation and degree of matrix-contactside localization of actinin-1 (d) or cortactin (e). a-c MannWhitney U test. d, e Spearman's correlation coefficient by rank test. 

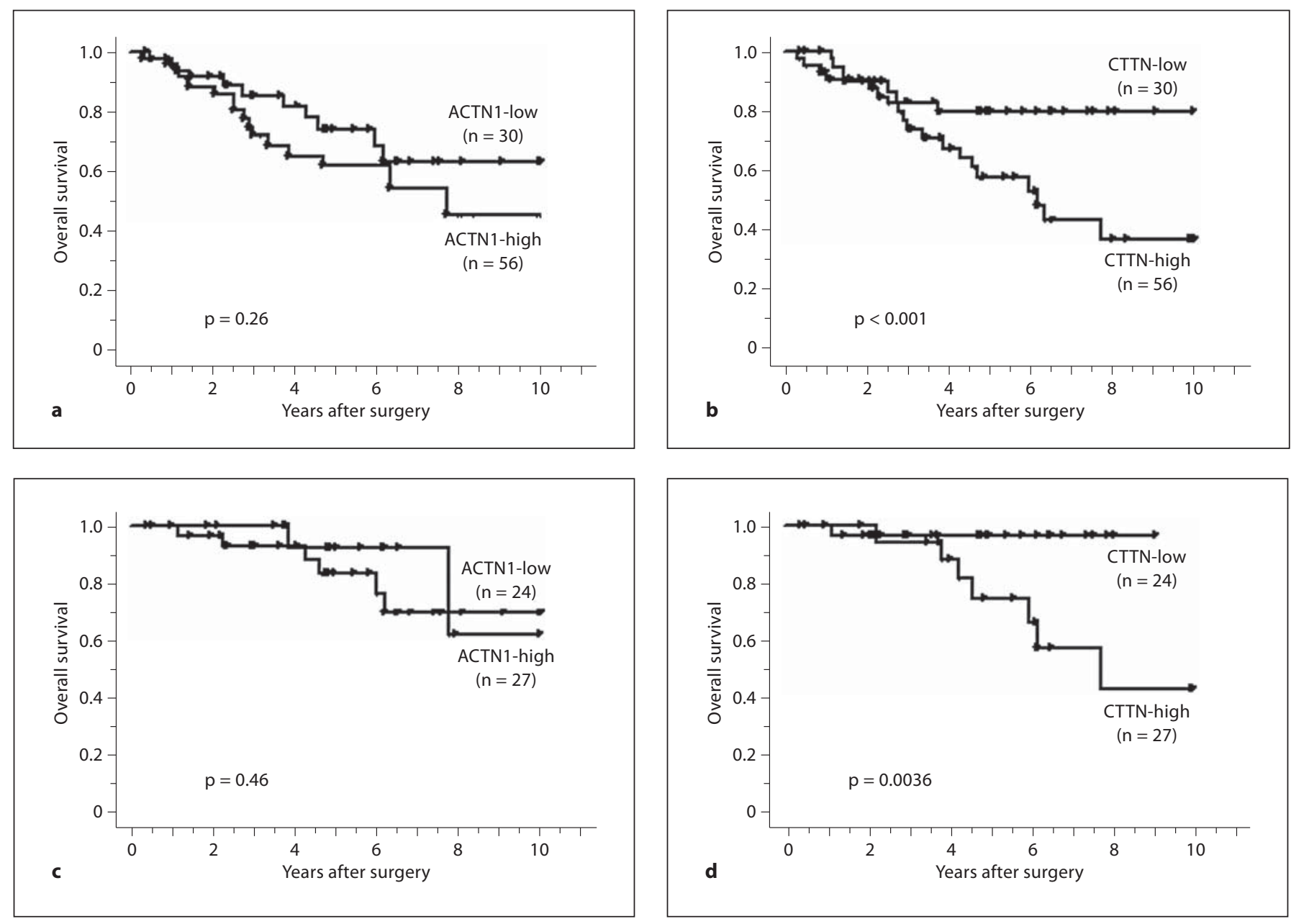

Fig. 7. Tumor-specific Kaplan-Meier survival curves for the matrix-contact-side localization of actinin-1 (ACTN1) and cortactin (CTTN) 'high' and 'low' groups. p values were calculated using a log-rank test. a, b 86 patients in stage I-III. c, d 51 patients in stage I.

ing actin fibers and linking transmembrane proteins to the actin cytoskeleton $[32,33]$. In the migrating cultured cells, actinin-1 is localized at the leading edge, a frontside cellular protrusion composed of filopodia and lamellipodia where filamentous and branched actin fibers are elongated and matrix-contact sites are assembled [34,35]. Both cortactin and VASP, which showed similar matrixcontact-side localization, also localize to the leading edge and participate in the stabilization of branching points and barbed-end elongation of actin filaments, respectively $[36,37]$. Another colocalization site of actinin-1, cortactin, and VASP is the invadopodium of cancer cells, which is formed by similar actin polymerization. Thus, matrix-contact-side localization of actinin-1 accompanied by cortactin, VASP, and actin in adenocarcinoma cells suggests the active assembly of actin filaments and the extension of cytoplasmic protrusions at matrix-contact sites.

Electron-microscopic findings provided evidence of cytoplasmic protrusions, with actinin-1 localization in the matrix-contact side of adenocarcinoma cells. Such cytoplasmic protrusions, approximately $0.5-1 \mu \mathrm{m}$ in size, were described previously as pseudopodial protrusions of squamous cell carcinoma cells [38, 39]. Extension of cytoplasmic protrusions in the direction of migration is considered to be an initial step in cancer cell invasion [21, 40]. Matrix-contact-side localization of actinin-1, cortactin, and VASP probably participates in the process of cancer cell invasion through the extension of cytoplasmic protrusions and subsequent cell migration. 

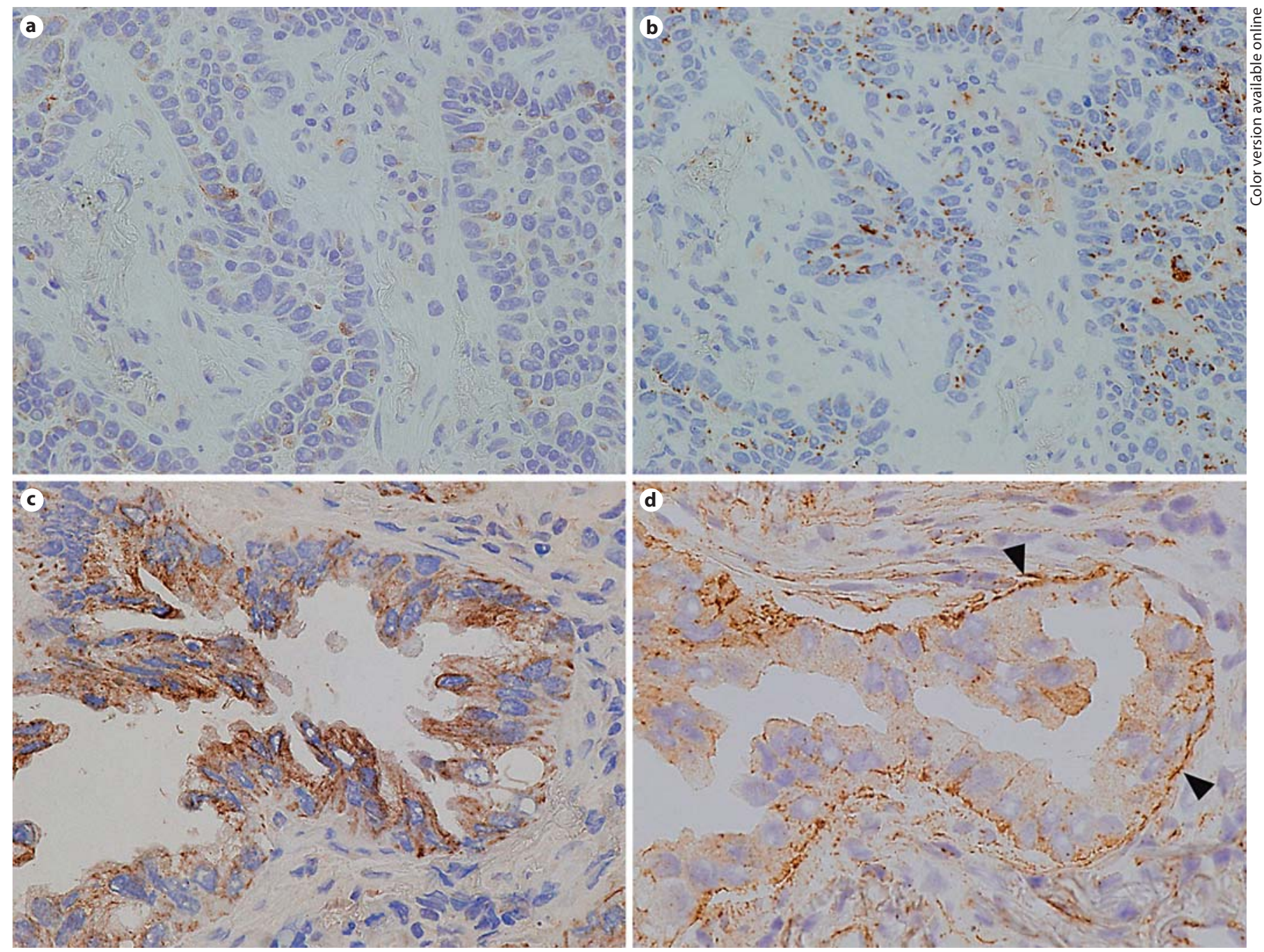

Fig. 8. Immunohistochemical expression of Ect2 (a, c) and actinin-1 (b, d) in lung adenocarcinomas using frozen sections. In BAC cells, neither cytoplasmic expression of Ect2 nor matrix-contact-side localization of actinin-1 is found (a, b). In papillary adenocarcinoma cells, Ect2 is expressed in cytoplasm and actinin-1 (arrowheads) is found at matrix-contact-side cytoplasm (c, d). Magnification: $\times 200$.

Matrix degradation is found at both the peripheral leading edge of migrating cells [6] and the ventral-side invadopodia of cultured cancer cells [3]. The close relationship between matrix-contact-side localization of actinin-1 and loss of basement membrane laminin-511 in our study suggests that invadopodia-related proteins also play significant roles in exaggerated matrix degradation in cancer cells.

Previous studies described that overexpression of cortactin mRNA was positively correlated with its translocation into matrix-contact sites in cultured breast cancer cells [41]. Although cortactin overexpression is found in several kinds of carcinomas [9, 42, 43], cortactin mRNA was reduced in lung adenocarcinomas. Expression of mRNA of other invadopodia-related proteins including actinin-1 was also reduced in adenocarcinomas. Actinin-1 is distinct from actinin-4, a nonmuscle isoform of $\alpha$-actinin which is overexpressed in colonic and pancreatic cancers $[44,45]$ and does not localize at focal adhesions [46]. Accordingly, it is considered that subcellular localization of invadopodia-related proteins may be regulated by other factors than their translation levels in lung adenocarcinomas. 
EGF stimulation promotes both leading-edge protrusion and invadopodia formation [21,30]; however, the incidence of EGFR-activating mutations was not significant in the cases of matrix-contact-side localization of actinin-1, and its effect was considered to be limited.

Correlation between immunohistochemical expression of Ect2 and matrix-contact-side localization of actinin-1 suggests the possibility that Ect 2 might regulate subcellular localization of invadopodia-related proteins in lung adenocarcinoma cells. Ect2 is a guanine nucleotide exchange factor for Rho family GTPases RhoA, Racl, and $\mathrm{CDC} 42$, localizes at nucleus, and participates in cytokinesis [47]. Ect2 is often overexpressed in non-small cell lung cancers, translocated to the cytoplasm, and participates in proliferation, invasion, and polarity abnormality through activation of Racl and actin remodeling $[48,49]$. Because Racl activation promotes both leading edge elongation [50] and invadopodia formation [29] through IRSp53/N-WASP/ARP2/3 complex formation, cytoplasmic expression of Ect 2 can also explain localization of invadopodia-related proteins to matrix-contactside cytoplasm.

Another hypothesis is that subcellular localization of actin-associated proteins is regulated by their subcellular localization of mRNA which is dependent on both microtubules and actin filament-based motors [51]. Further studies using fluorescent in situ hybridization might clarify whether subcellular localization of invadopodia-associated proteins is determined at the protein level or the RNA level.

Our results suggest the clinical utility of subcellular localization of invadopodia-related proteins to predict metastasis and prognosis of lung adenocarcinomas. Invadopodia-related proteins as well as laminin might also be useful for the discrimination of subtypes, especially to discern whether the lesion is a noninvasive bronchioloalveolar subtype or an invasive subtype.

This investigation exclusively focused on lung adenocarcinomas; however, our preliminary study concerning to lung squamous neoplasms revealed that matrix-contact-side localization of both actinin-1 and cortactin was found in invasive squamous cell carcinomas, but not in their precursor lesions, squamous metaplasia or carcinoma in situ (online suppl. fig. 3). Accordingly, it is possible to say that invadopodia-related proteins play similar roles in the invasion of types of cancers other than adenocarcinomas.

In conclusion, our studies revealed matrix-contactside localization of invadopodia-related proteins, actinin-1 and cortactin, in the lung adenocarcinoma cells and their correlation with invasion, metastasis, and poor prognosis. Ect2 was one possible regulator of matrix-contact-side localization of invadopodia-related proteins.

\section{Acknowledgements}

The authors thank Kei Itou, Makiko Iida, Masaki Sekine, Keisuke Kitagaki, Takashi Sekiya, and Noriko Katsuta for their technical assistance. This research was supported by a grant-inaid from the Ministry of Education, Science, Sports and Culture of Japan.

\section{References}

1 Critchley DR: Focal adhesions - the cytoskeletal connection. Curr Opin Cell Biol 2000;12:133-139.

-2 Lauffenburger DA, Horwitz AF: Cell migration: a physically integrated molecular process. Cell 1996;84:359-369.

3 Buccione R, Orth JD, McNiven MA: Foot and mouth: podosomes, invadopodia and circular dorsal ruffles. Nat Rev Mol Cell Biol 2004;5:647-657.

4 Clark ES, Whigham AS, Yarbrough WG, et al: Cortactin is an essential regulator of matrix metalloproteinase secretion and extracellular matrix degradation in invadopodia. Cancer Res 2007;67:4227-4235.
5 Artym VV, Zhang Y, Seillier-Moiseiwitsch F, et al: Dynamic interactions of cortactin and membrane type 1 matrix metalloproteinase at invadopodia: defining the stages of invadopodia formation and function. Cancer Res 2006;66:3034-3043.

-6 Takino T, Saeki H, Miyamori H, et al: Inhibition of membrane-type 1 matrix metalloproteinase at cell-matrix adhesions. Cancer Res 2007:67:11621-11629.

7 Wolf K, Friedl P: Mapping proteolytic cancer cell-extracellular matrix interfaces. Clin Exp Metastasis 2009;26:289-298.

-8 Gibcus JH, Mastik MF, Menkema L, et al: Cortactin expression predicts poor survival in laryngeal carcinoma. Br J Cancer 2008; 98 : 950-955.
-9 Tsai WC, Jin JS, Chang WK, et al: Association of cortactin and fascin-1 expression in gastric adenocarcinoma: correlation with clinicopathological parameters. J Histochem Cytochem 2007;55:955-962.

10 Iguchi T, Aishima S, Taketomi A, et al: Fascin overexpression is involved in carcinogenesis and prognosis of human intrahepatic cholangiocarcinoma: immunohistochemical and molecular analysis. Hum Pathol 2009;40:174-180.

11 Dertsiz L, Ozbilim G, Kayisli Y, et al: Differential expression of VASP in normal lung tissue and lung adenocarcinomas. Thorax 2005;60:576-581. 
12 Semba S, Iwaya K, Matsubayashi J, et al: Coexpression of actin-related protein 2 and Wiskott-Aldrich syndrome family verproline-homologous protein 2 in adenocarcinoma of the lung. Clin Cancer Res 2006;12: 2449-2454

13 Watanabe N, Nakajima I, Abe S, et al: Staining pattern of type IV collagen and prognosis in early stage adenocarcinoma of the lung. J Clin Pathol 1994;47:613-615.

14 Matsui K, Kitagawa M, Sugiyama S, et al: Distribution pattern of the basement membrane components is one of the significant prognostic correlates in peripheral lung adenocarcinomas. Hum Pathol 1995;26:186194.

15 Akashi T, Ito E, Eishi Y, et al: Reduced expression of laminin alpha 3 and 28 alpha 5 chains in non-small cell lung cancers. Jpn J Cancer Res 2001;92:293-301.

16 Akashi T, Minami J, Ishige Y, et al: Basement membrane matrix modifies cytokine interactions between lung cancer cells and fibroblasts. Pathobiology 2005;72:250-259.

- 17 Soini Y, Paakko P, Autio-Harmainen H: Genes of laminin B1 chain, alpha 1 (IV) chain of type IV collagen, and 72-kd type IV collagenase are mainly expressed by the stromal cells of lung carcinomas. Am J Pathol 1993;142:1622-1630.

- 18 Kanomata N, Nakahara R, Oda T, et al: Expression and localization of mRNAs for matrix metalloproteinases and their inhibitors in mixed bronchioloalveolar carcinomas with invasive components. Mod Pathol 2005 $18: 828-837$

19 Kawano N, Osawa H, Ito T, et al: Expression of gelatinase A, tissue inhibitor of metalloproteinases-2, matrilysin, and trypsin(ogen) in lung neoplasms: an immunohistochemical study. Hum Pathol 1997;28:613-622.

20 Tokuraku M, Sato H, Murakami S, et al: Activation of the precursor of gelatinase A/72 $\mathrm{kDa}$ type IV collagenase/MMP-2 in lung carcinomas correlates with the expression of membrane-type matrix metalloproteinase (MT-MMP) and with lymph node metastasis. Int J Cancer 1995;64:355-359.

-21 Yamaguchi H, Lorenz M, Kempiak S, et al: Molecular mechanisms of invadopodium formation: the role of the N-WASP-Arp2/3 complex pathway and cofilin. J Cell Biol 2005; 168:441-452.

22 Linder S: The matrix corroded: podosomes and invadopodia in extracellular matrix degradation. Trends Cell Biol 2007;17:107117.

23 Cortesio CL, Chan KT, Perrin BJ, et al: Calpain 2 and PTP1B function in a novel pathway with Src to regulate invadopodia dynamics and breast cancer cell invasion. J Cell Biol 2008;180:957-971.

Subcellular Localization of Actinin-1 and Cortactin
24 Travis WD, Brambilla E, Muller-Hermelink $\mathrm{H}$, et al: Tumors of the Lung, Pleura, Thymus and Heart. World Health Organization Classification of Tumors. Pathology and Genetics. Lyon, IARC, 2004, pp 35-44.

25 Motoi N, Szoke J, Riely GJ, et al: Lung adenocarcinoma: modification of the $2004 \mathrm{WHO}$ mixed subtype to include the major histologic subtype suggests correlations between papillary and micropapillary adenocarcinoma subtypes, EGFR mutations and gene expression analysis. Am J Surg Pathol 2008;32: 810-827.

26 Goldstraw P: Staging Manual in Thoracic Oncology. Orange Park, Editorial Rx Press, 2009.

27 Rosario CO, Ko MA, Haffani YZ, et al: Plk4 is required for cytokinesis and maintenance of chromosomal stability. Proc Natl Acad Sci USA 2010;107:6888-6893.

28 Shedden K, Taylor JM, Enkemann SA, et al: Gene expression-based survival prediction in lung adenocarcinoma: a multi-site, blinded validation study. Nat Med 2008;14:822827.

29 Muralidharan-Chari V, Hoover H, Clancy J, et al: ADP-ribosylation factor 6 regulates tumorigenic and invasive properties in vivo. Cancer Res 2009;69:2201-2209.

30 Chan AY, Raft S, Bailly M, et al: EGF stimulates an increase in actin nucleation and filament number at the leading edge of the lamellipod in mammary adenocarcinoma cells. J Cell Sci 1998;111:199-211.

31 Waites GT, Graham IR, Jackson P, et al: Mutually exclusive splicing of calcium-binding domain exons in chick alpha-actinin. J Biol Chem 1992;267:6263-6271.

32 Burridge K, Feramisco JR: Non-muscle alpha actinins are calcium-sensitive actinbinding proteins. Nature 1981;294:565-567.

33 Otey CA, Carpen O: Alpha-actinin revisited: a fresh look at an old player. Cell Motil Cytoskeleton 2004;58:104-111.

34 Laukaitis CM, Webb DJ, Donais K, et al: Differential dynamics of alpha 5 integrin, paxillin, and alpha-actinin during formation and disassembly of adhesions in migrating cells. J Cell Biol 2001;153:1427-1440.

35 Mattila PK, Lappalainen P: Filopodia: molecular architecture and cellular functions. Nat Rev Mol Cell Biol 2008;9:446-454.

-36 Weaver AM, Karginov AV, Kinley AW, et al: Cortactin promotes and stabilizes Arp2/3induced actin filament network formation. Curr Biol 2001;11:370-374.

-37 Krause M, Bear JE, Loureiro JJ, et al: The Ena/VASP enigma. J Cell Sci 2002;115:47214726.

38 Takubo K, Nishimura H, Taniguchi Y, et al: Junctions between intraepithelial carcinoma and non-neoplastic tissue of the esophagus. Light and electron microscopic studies. Acta Pathol Jpn 1984;34:785-796.
39 White FH, Gohari K: A quantitative study of lamina densa alterations in hamster cheek pouch carcinogenesis. J Pathol 1981;135: 277-294.

40 Friedl P, Wolf K: Tumour-cell invasion and migration: diversity and escape mechanisms. Nat Rev Cancer 2003;3:362-374.

41 van Damme H, Brok H, Schuuring-Scholtes $\mathrm{E}$, et al: The redistribution of cortactin into cell-matrix contact sites in human carcinoma cells with 11q13 amplification is associated with both overexpression and posttranslational modification. J Biol Chem 1997;272:7374-7380.

42 Rodrigo JP, Garcia-Carracedo D, Garcia LA, et al: Distinctive clinicopathological associations of amplification of the cortactin gene at 11 q13 in head and neck squamous cell carcinomas. J Pathol 2009;217:516-523.

43 Hui R, Ball JR, Macmillan RD, et al: EMS1 gene expression in primary breast cancer: $\mathrm{re}$ lationship to cyclin D1 and oestrogen receptor expression and patient survival. Oncogene 1998;17:1053-1059.

-44 Honda K, Yamada T, Hayashida Y, et al: Actinin- 4 increases cell motility and promotes lymph node metastasis of colorectal cancer. Gastroenterology 2005;128:51-62.

$\checkmark 45$ Kikuchi S, Honda K, Tsuda H, et al: Expression and gene amplification of actinin- 4 in invasive ductal carcinoma of the pancreas. Clin Cancer Res 2008;14:5348-5356.

$\checkmark 46$ Honda K, Yamada T, Endo R, et al: Actinin-4, a novel actin-bundling protein associated with cell motility and cancer invasion. J Cell Biol 1998;140:1383-1393.

-47 Fields AP, Justilien V: The guanine nucleotide exchange factor (GEF) Ect2 is an oncogene in human cancer. Adv Enzyme Regul 2010;50:190-200.

48 Hirata D, Yamabuki T, Miki D, et al: Involvement of epithelial cell transforming sequence- 2 oncoantigen in lung and esophageal cancer progression. Clin Cancer Res 2009; 15:256-266.

49 Justilien V, Fields AP: Ect2 links the PKCiota-Par6alpha complex to Racl activation and cellular transformation. Oncogene 2009;28: 3597-3607.

50 Takenawa T, Miki H: WASP and WAVE family proteins: key molecules for rapid rearrangement of cortical actin filaments and cell movement. J Cell Sci 2001;114:18011809.

51 Mingle LA, Okuhama NN, Shi J, et al: Localization of all seven messenger RNAs for the actin-polymerization nucleator Arp2/3 complex in the protrusions of fibroblasts. J Cell Sci 2005; 118:2425-2433. 